\title{
cmaJOPEN
}

\section{Amoxicillin oral provocation challenge in a primary care clinic: a descriptive analysis}

\author{
Derek Paul Gateman MD, Jessie Erin Rumble MScN, Jennifer L.P. Protudjer PhD, Harold Kim MD
}

Abstract

Background: Penicillin is the most frequently reported drug allergen; however, most of these allergies are not true allergies and do not justify the prescription of alternative, less effective and more expensive antibiotic drugs. We aimed to show that patients at low risk of amoxicillin allergy can safely and efficiently undergo oral provocation challenge (OPC) by their primary care physician.

Methods: In this descriptive analysis, we conducted a retrospective chart review of all primary care patients who had undergone OPC from November 2017 to October 2019 in the Amoxicillin Allergy Clinic at the North Perth Family Health Team, Listowel, Ontario. Eligibility for OPC among patients 18 months and older was determined through review of a self-reported patient intake form asking about symptoms, onset, duration, history and family history of allergic reactions, as well as the patient's electronic medical record. Patients were considered to be at low risk of true penicillin allergy if there was no history of anaphylaxis or severe cutaneous reactions. Those with low-risk allergic reactions returned for testing with an OPC to amoxicillin. We collected data on clinical characteristics, antibiotic exposure, parental drug allergy, response to OPC and wait time from referral. We used $t$ tests to describe and compare these variables. Our primary outcome was reaction to OPC by severity as categorized by the World Allergy Organization grading system. Our secondary outcome was the time from referral to completed testing.

Results: In total, we included 99 patients (mean age 28.3, standard deviation [SD] 21.2 yr); 72 (73\%) were female. Of those tested, $97 \%(n=96)$ completed the OPC with no reaction, $3 \%(n=3)$ had mild immediate reactions, and no serious immediate reactions developed. Mean wait time to testing was 59.0 (SD 69.8) days, with a median (interquartile range) of 39.5 (13.5-70.0) days.

Interpretation: Oral provocation challenge presents a safe and accessible opportunity for primary care providers to address erroneous allergy labels to penicillin and related drugs within the primary care office setting. There could be positive public health implications if OPC to penicillin drugs is implemented in primary care.

ffecting about $10 \%$ of patients, penicillin drug allergy is the most frequently reported drug allergy. ${ }^{1}$ Penicillin drug allergy is commonly diagnosed after a childhood rash is assumed to be caused by recent penicillin administration but which is not investigated further. ${ }^{2}$ T-lymphocyte-mediated nonimmediate reactions typically present with maculopapular rashes days after ingestion. ${ }^{3}$ In contrast, immunoglobulin E ( $\mathrm{IgE}$ )-mediated immediate reactions occur within the first hour and appear as urticaria, angioedema, rhinitis, bronchospasm or, rarely, as anaphylaxis. ${ }^{2,4}$

Despite their clinical use for over 70 years, penicillin and penicillin-related antibiotics continue to be recommended for first-line therapy in numerous respiratory tract, skin, joint and cardiovascular infections. ${ }^{5,6}$ Although most reactions following use of penicillin and related antibiotics are not true drug allergies, medical practitioners often prescribe less effective or more expensive alternatives. ${ }^{7-9}$ This leads to an increased risk of adverse events, more drug-resistant organisms and more Clostridium difficile infections. ${ }^{10,11}$
Presently, primary care providers struggle to rule out penicillin drug allergies. ${ }^{12} \mathrm{~A}$ referral to an allergist for all patients with suspected penicillin drug allergy is impractical, as there are millions of patients with presumed penicillin allergy. ${ }^{13} \mathrm{In}$ Canada, there is less than 1 allergist per 100000 people. Not surprisingly, this translates to long wait times, with an estimated wait time for penicillin drug allergy consultation of 12-18 months in rural southwestern Ontario (H.K., unpublished observations, 2019).

Primary care providers have limited experience and training in performing skin testing for drug allergy or measurement of specific IgE levels. Skin testing can be inconvenient and time

\section{Competing interests: None declared.}

This article has been peer reviewed.

Correspondence to: Derek Gateman, derek.gateman@medportal.ca CMAJ Open 2021. DOI:10.9778/cmajo.20200077 
consuming, and offers a poor clinical predictive value for nonimmediate reactions. ${ }^{2}$ However, oral provocation challenge (OPC) testing presents an alternative that has been shown to be safe in both pediatric and adult populations without the need for allergy skin testing. ${ }^{2,14}$ Oral provocation challenge is the definitive test for penicillin allergy. ${ }^{2}$ When a patient with possible allergy can tolerate a reasonable dose of the presumed allergen in an OPC, allergy is essentially ruled out. ${ }^{2}$ Thus, we aimed to show that patients with a low risk of amoxicillin allergy can safely and efficiently undergo OPC by their primary care providers.

\section{Methods}

\section{Study design and setting}

We conducted a descriptive analysis of cross-sectional data for patients who underwent consultation and OPC for presumed allergy to penicillin or related antibiotics between November 2017 to October 2019, in the primary care Amoxicillin Allergy Clinic at the rural North Perth Family Health Team in Listowel, Ontario (Appendix 1, available at www. cmajopen.ca/content/9/2/E394/suppl/DC1). The clinic is typically held monthly.

Initially, consultations and OPC were performed in the Listowel Memorial Hospital outpatient department from November 2017 to January 2018, before moving to the family medicine office at the North Perth Family Health Team in February 2018.

\section{Participants}

Patients who were allergic to amoxicillin or penicillin were recruited for the Amoxicillin Allergy Clinic after they returned a patient intake form that had been distributed through the North Perth Family Health Team office, the Listowel Memorial Hospital emergency department and preoperative clinic, and a local dentist's office. The form included the patient's history of anaphylaxis; allergic reaction symptoms, onset and duration; and personal and first-degree relatives' medical histories (Appendix 2, available at www.cmajopen.ca/content/9/2/E394/ suppl/DC1). We determined eligibility of patients who had returned the form to undergo OPC through review of their electronic medical record from their family physician and patient consultation. At the consultation appointment, we interviewed the patient to clarify their reaction type as immediate or nonimmediate, determine reaction symptoms and their severity, and review their medical history.

Patients were eligible for OPC if they were 18 months of age or older, and if they were considered to be at low risk of true penicillin allergy (i.e., no history of anaphylaxis or severe cutaneous reactions). Patients were not eligible for OPC if they had previously received penicillin without any reaction. They were also not eligible if their reaction was IgE-mediated (e.g., angioedema, bronchospasm or anaphylaxis), if they had a previous severe reaction (e.g., Stevens-Johnson syndrome, toxic epidermal necrolysis, acute generalized exanthematous pustulosis or a drug reaction with eosinophilia and systemic symptoms), or if their clinical notes or history were substan- tially inconsistent or incomplete (Figure 1). Patients taking concurrent medications were not excluded.

\section{Oral provocation challenge protocol}

If patients were deemed low risk of true penicillin allergy after consultation and clinical history review, they were offered graded OPC immediately in either the Listowel Memorial Hospital outpatient clinic (November 2017 to January 2018) or the North Perth Family Health Team office (from February 2018). Patient consent was obtained before the procedure. Both settings were equipped with diphenhydramine elixir to manage mild reactions and epinephrine to manage anaphylactic reactions, as well as standard resuscitation medications and equipment. ${ }^{15}$

A 2-step graded OPC to amoxicillin was performed by a family physician and nurse practitioner. Amoxicillin was supplied as an oral suspension $(250 \mathrm{mg} / 5 \mathrm{~mL})$, with a total challenge dose of $500 \mathrm{mg}$ for patients weighing more than $10 \mathrm{~kg}$, or $45 \mathrm{mg} / \mathrm{kg}$ for pediatric patients weighing less than $10 \mathrm{~kg}$. 2,16 The challenge dose was expected to be equivalent to that of a single therapeutic dose.

Patients were first administered $10 \%$ of the challenge dose $(50 \mathrm{mg}$ or $4.5 \mathrm{mg} / \mathrm{kg}$ if the patient weighed $<10 \mathrm{~kg}$ ) and were observed for 20 minutes. If there was no reaction, patients received $90 \%$ of the therapeutic dose $(450 \mathrm{mg}$ or $40.5 \mathrm{mg} / \mathrm{kg}$ if the patient weighed $<10 \mathrm{~kg}$ ) and were observed for 1 hour. If patients did not react to the therapeutic dose, this allergy was removed from the patient's chart, and the patient was advised to inform their pharmacist. This protocol was developed based on methods reported in publications by Mill and colleagues, ${ }^{2}$ Hjortlund and colleagues ${ }^{17}$ and Labrosse and colleagues. ${ }^{18}$

We considered the reaction to be positive on the development of objective findings (e.g., urticaria, wheeze, swelling); we did not consider subjective symptoms (e.g., pruritus without skin changes, dizziness) alone to be sufficient to diagnose an immune reaction.

We provided patients with follow-up instructions in writing that were adapted from the American College of Allergy, Asthma and Immunology. ${ }^{19}$ Patients were instructed to report any delayed symptoms of rash, hives, wheezing or swelling in the same day or week, or during any subsequent treatments. Patients were advised about the safety of future prescription of penicillin, if clinically indicated.

\section{Data sources}

We retrospectively reviewed patient charts from the Amoxicillin Allergy Clinic for patients seen in the clinic from November 2017 to October 2019. The review included patient intake forms, consultation notes and clinic notes related to OPC. Data abstraction was performed by a single assessor (D.P.G) using a predefined abstraction form.

We selected variables based on a previously published study from a specialist clinic. ${ }^{2}$ These included data on demographic and clinical characteristics, previous antibiotic exposure, personal and first-degree relatives' medical histories, reaction management and response to OPC. 


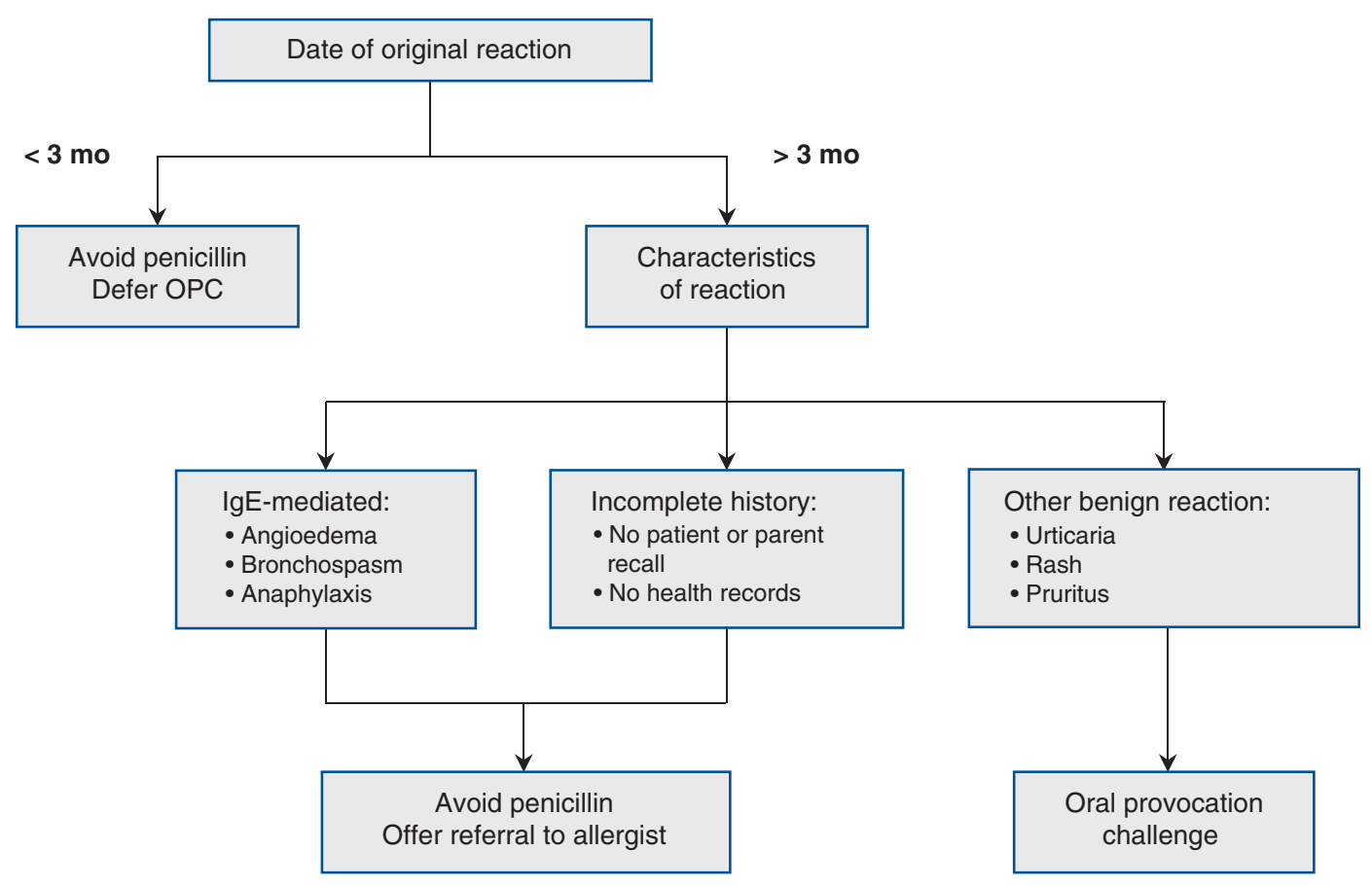

Figure 1: Protocol for eligibility for oral provocation challenge, using the self-reported results from the patient intake form (Appendix 2, available at www.cmajopen.ca/content/9/2/E394/suppl/DC1).

\section{Outcomes}

Our primary outcome of interest was the frequency and type of reaction to OPC. We classified the OPC reactions using the World Allergy Organization Subcutaneous Immunotherapy Systemic Reaction Grading System, which comprises 5 grades ranging from 1 (cutaneous, upper respiratory, conjunctival or other symptoms) to 5 (death; Table 1 ). ${ }^{20}$ For patients with reaction to OPC, demographic information and reaction were described narratively. Our secondary outcome was the wait time to complete OPC.

Variables included sex (binary, male or female), age (continuous, yr) and wait times (continuous, from day of review of the patient intake form to the date of testing). We gave additional consideration to pediatric patients by stratifying age into less than 18 years and 18 years and older.

Wait times were stratified into 2 groups: less than 45 days and more than 45 days. We thought that a wait time of more than 6 weeks would be perceived by patients as a subjectively long wait versus a short wait in the context of elective allergy testing. To clarify the timing, the wait time began with receipt of the completed intake survey and ended with the consultation and provision of OPC (simultaneous) in the clinic.

Additional variables included history of immediate allergy and family history of penicillin allergy. We considered reactions within 1 hour of drug exposure to be immediate. ${ }^{2} \mathrm{We}$ defined a family history of penicillin allergy as any report of a known parental allergy, regardless of severity. For patients with reaction to OPC, demographic information and reaction were described narratively.

\section{Statistical methods}

We used sample size, percentages and means with standard deviations (SDs) to describe data (e.g., sex, age, average wait

\begin{tabular}{|l|l|}
\hline $\begin{array}{l}\text { Table 1: World Allergy Organization } \\
\text { Subcutaneous Immunotherapy Systemic } \\
\text { Reaction Grading System }{ }^{20}\end{array}$ \\
\hline Grade & \multicolumn{1}{c|}{ Symptoms or signs } \\
\hline 1 & $\begin{array}{l}\text { Cutaneous, upper respiratory (rhinitis, } \\
\text { throat clearing, cough), conjunctival or } \\
\text { other (nausea, metallic taste or } \\
\text { headache) }\end{array}$ \\
\hline 2 & $\begin{array}{l}\text { More than 1 organ system, lower } \\
\text { respiratory, gastrointestinal, or uterine } \\
\text { cramps }\end{array}$ \\
\hline 3 & $\begin{array}{l}\text { Lower respiratory or upper respiratory } \\
\text { (laryngeal, uvula or tongue edema) }\end{array}$ \\
\hline 4 & $\begin{array}{l}\text { Respiratory failure or cardiovascular } \\
\text { (hypotension) }\end{array}$ \\
\hline 5 & Death \\
\hline
\end{tabular}


times and family and personal history of penicillin allergy, including reaction and reaction grade). We compared differences in average wait times between pediatric and adult patients using $t$ tests. We also calculated medians with interquartile ranges (IQRs) and maximums wait times. We used the Wilson method to calculate $95 \%$ confidence intervals (CIs) for the proportion of patients with a reaction. Observations with missing information on variables were excluded from the relevant analyses. Statistical significance was set at $p<0.05$. Data were analyzed using Stata 15.1.

\section{Ethics approval}

We obtained approval for this study from the Hamilton Integrated Research Ethics Board, affiliated with McMaster University (Project No. 7414-C).

\section{Results}

Between Nov. 20, 2017, and Oct. 1, 2019, we offered OPC to 103 patients. Of the 99 (97\%) who completed OPC, 72 were female $(73 \%)$, with a mean age of 28.3 (SD 21.1) years. In total, $41.8 \%$ (41/98) were pediatric patients (mean age 8.0 [SD 4.0] yr) (Table 2). Mean age for adult patients was 42.9 (SD 15.5) years. Although the sex distribution was nearly even in the pediatric sample (19 male, 22 female), the adult sample population was disproportionately female (50/58).

The mean wait time was about 2 months (59.0 [SD 69.8] d), with median and maximum wait times of 39.5 (IQR 13.5$70.0)$ and 420 days, respectively. Mean wait times were significantly longer for pediatric patients compared with those for adult patients (77.3 [SD 90.2] v. 45.3 [SD 45.6] d; $p<0.02)$. Corresponding median and maximum wait times were, for pediatrics, 47 (IQR 18-94) and 420 days, respectively, and for adults, 39 (IQR 12-59) and 201 days, respectively.

When known, most patients had neither a family history of penicillin allergy $(82.5 \%, 80 / 97)$ nor a personal history of immediate reaction to penicillin $(90.0 \%, 81 / 90)$.

Reactions to OPC were uncommon (3/99, 3.0\%, $95 \%$ CI $3 \%-9 \%)$. The patients who had an immediate reaction were pediatric and had a World Allergy Organization reaction grade of 1 . All 3 patients who reacted had a personal history of immediate reaction, out of the 9 patients with a similar history (reaction rate of $33 \%$ ). Diphenhydramine was used to treat symptoms; epinephrine was not administered in any cases. There were no reported delayed reactions to OPC. The demographic characteristics of the patients who reacted to the challenge and our narrative descriptions of their reactions to amoxicillin are provided in Appendix 3, available at www.cmajopen.ca/content/9/2/E394/suppl/DC1.

No associations were identified for family history of penicillin allergy when we compared pediatric with adult patients. Similarly, we did not find associations between family history and an interaction between sex and age. There was no difference in wait times whether patients had or did not have a family history of penicillin allergy. We could not determine associations between demographic characteristics and a personal history of immediate penicillin reaction.

\section{Table 2: Demographic characteristics of patients who were} included in the study

\begin{tabular}{|c|c|}
\hline Characteristic & $\begin{array}{c}\text { No. (\%) of included patients }{ }^{*} \dagger \\
\qquad n=99\end{array}$ \\
\hline \multicolumn{2}{|l|}{ Sex } \\
\hline Male & $27(27)$ \\
\hline Female & $72(73)$ \\
\hline Age, yr; mean \pm SD $(n=98)$ & $28.3 \pm 21.1$ \\
\hline Age $<18 \mathrm{yr}$ & $41(42)$ \\
\hline Mean & $8.0 \pm 4.1$ \\
\hline Age $\geq 18 \mathrm{yr}$ & $57(58)$ \\
\hline Mean & $42.9 \pm 15.5$ \\
\hline Wait time, d; mean \pm SD $(n=97)$ & $59.0 \pm 69.8$ \\
\hline Wait time $<45 d$ & $53(54)$ \\
\hline Mean & $18.0 \pm 13.8$ \\
\hline Wait time $\geq 45 \mathrm{~d}$ & $46(44)$ \\
\hline Mean & $108.4 \pm 78.0$ \\
\hline \multicolumn{2}{|c|}{ Family history of penicillin allergy $(n=97)$} \\
\hline No & $80(83)$ \\
\hline Yes & $17(17)$ \\
\hline \multicolumn{2}{|c|}{ Personal history of immediate reaction to penicillin $(n=90)$} \\
\hline No & $81(90)$ \\
\hline Yes & $9(10)$ \\
\hline \multicolumn{2}{|c|}{$\begin{array}{l}\text { Note: } \mathrm{SD}=\text { standard deviation. } \\
\text { *Unless specified otherwise. } \\
\text { †Observations with missing information on a characteristic were excluded from } \\
\text { the relevant analysis. }\end{array}$} \\
\hline
\end{tabular}

\section{Interpretation}

In our study, only 3 patients with a history of mild reactions had reactions to OPC, which were minor. Each of the 3 reactions were grade 1 , meaning limited to just 1 sign or symptom (cutaneous, upper respiratory, conjunctival or other). Diphenhydramine was used to treat symptoms; epinephrine was not administered in any cases. There were no reported delayed reactions to OPC in our study.

Anaphylaxis with penicillin OPC in patients at low risk is uncommon and rarely fatal. ${ }^{219-21}$ By comparison, subcutaneous immunotherapy, which is commonly performed in primary care, has a similar risk of systemic adverse events. ${ }^{22,23}$ The training, equipment and skills to manage these complications would be directly relatable to OPC reactions. Given the high prevalence of low-risk penicillin allergy in Canada and long wait times for access to specialist testing, primary care providers may benefit from having a safe and accessible testing option.

This study adds to the body of evidence evaluating the safety of OPC for low-risk penicillin and amoxicillin allergy. Immediate mild allergic reactions occurred in 3\% of patients in our study, which is similar to that reported by Labrosse and 
colleagues. ${ }^{18}$ Notably, that study reported an immediate allergic reaction rate of $2.3 \%$ during 5 -day OPC in a pediatric population with mean age of 5.1 years. ${ }^{20}$ In another study in a younger population (median age 1.7 [IQR 1.0-3.9] yr), reaction rates were higher $(5.9 \%) .^{2}$ Although the methodology was different (patients were tested in an allergy clinic and had preceding intracutaneous testing), Hjortlund and colleagues ${ }^{17}$ found a reaction rate of $4.5 \%(15 / 335)$ to OPC with penicillin V. ${ }^{19}$ Sundquist and colleagues ${ }^{12}$ also performed skin testing in an allergy clinic before OPC, and none of the 36 adult participants had an immediate reaction. Iammatteo and colleagues ${ }^{14}$ conducted a single blind placebo-controlled direct amoxicillin challenge with 155 adults (mean age $51 \mathrm{yr}$ ), among whom the reaction rate was $2.6 \%$. In a small study of 20 adult outpatients, the OPC reaction rate was $0 \%$, and none had immediate or delayed hypersensitivity reactions. ${ }^{24}$ Our study supports current evidence for conducting OPC without skin testing and extends this to the primary care office setting.

There were 3 immediate allergic reactions among those 9 patients with a history of immediate reaction (reaction rate of $33 \%)$. This represents a 10 -fold increase over the reaction rate among all patients in our study who underwent OPC. Although history of immediate reaction is a risk factor for allergic reaction, most patients with a history of immediate reaction did not react to OPC, and were de-labelled as allergic to penicillin and related drugs.

In rural southwestern Ontario, a patient referred for penicillin drug allergy to an allergist commonly waits at least 12 months for assessment and testing. Patients in our sample population waited 59.0 days on average to be assessed, and $54 \%$ of patients were seen in less than 45 days. The allergy clinic was held once monthly; patients waited an average of 2 clinic cycles. There was a significantly longer wait in the pediatric population compared with that in adults, the reasons for which are unclear but may, in part, be due to school attendance or need for caregiver accompaniment. A study of physician barriers to patient recruitment noted that patients' unwillingness to take time to be tested was ranked most important. ${ }^{12}$

To add to the body of evidence evaluating the safety of OPC in primary care, our methodology could be replicated by primary care providers in different settings. A larger sample size would allow for the study of the effects of demographic characteristics, such as age, which may drive the study of specific mechanisms in reaction to OPC. Preoperative patients present another potential area of study. Although rapid access to OPC to de-label patients as allergic to penicillin drugs can be integrated into the surgical pathway, OPC by primary care providers may provide a cheaper or more convenient option. ${ }^{25} \mathrm{We}$ also propose to reassess the patients included in our study in the future to report which of these patients later received and tolerated penicillin for a clinical indication.

As the prevalence of inappropriate penicillin allergy labelling decreases, the prescribing of penicillin alternatives may decrease. We suggest that changing trends in antibiotic prescription may affect bacterial resistance patterns that could be studied. Subse- quently, there may be both reductions in cost to the health care system and improvements in patient outcomes., ${ }^{9,26}$

In addition to the potential medical and public health benefits we have outlined, the clinical approach used at the primary care Amoxicillin Allergy Clinic was straightforward and not costly. Most primary care practitioners should be able to implement penicillin OPC in their clinics quite easily at minimal or no extra cost to the health care system.

\section{Limitations}

The study sample was dependent on referral from local primary care providers, so we are unaware if patients with greater or lesser risk of low-risk reactions were included in the study. The intake form responses were patient-reported, introducing possible recall bias. The form was available only in English. The total number of intake forms distributed and number of excluded patients were not measured.

Chart review was conducted by a single abstractor; therefore, it is possible that errors may have occurred during data abstraction. The definition for wait times was based on the opinions of the authors, and not formally tested. The low reaction rate for OPC precluded investigation of underlying demographic characteristics. Furthermore, only $10 \%$ of patients reported a personal history of immediate penicillin reaction; therefore, we could not calculate associations with sex, age and wait times. Our sample population was not large enough to consider factors that would have predicted an OPC reaction. Although patients were instructed to follow up with the clinic, delayed reactions may have been missed.

Importantly, the study was set at a single centre with 1 physician assessing patient eligibility and administering the OPC protocol; errors may have occurred at various points in clinic processes, protocol administration and clinical documentation. While we believe that the eligibility criteria for OPC and the clinical approach used at the Amoxicillin Allergy Clinic should be able to be implemented in other settings, we recognize that expertise and comfort level in performing OPC may differ among other primary care physicians.

\section{Conclusion}

We found OPC in the primary care setting within the primary care office setting to be a safe and accessible option for primary care providers to address erroneous penicillin allergy labels. Widespread implementation of penicillin OPC by primary care providers could have major health implications, particularly in sparsely populated regions.

\section{References}

1. Warrington R, Silviu-Dan F, Wong T. Drug allergy. Allergy Astbma Clin Immunol 2018;14(Suppl 2):60.

2. Mill C, Primeau M-N, Medoff E, et al. Assessing the diagnostic properties of a graded oral provocation challenge for the diagnosis of immediate and nonimmediate reactions to amoxicillin in children. 7 AMA Pediatr 2016; 170:e160033.

3. Shenoy ES, Macy E, Rowe T, et al. Evaluation and management of penicillin allergy: a review. 7AMA 2019;321:188-99.

4. Gonzalez-Estrada A, Radojicic C. Penicillin allergy: a practical guide for clinicians. Cleve Clin 7 Med 2015;82:295-300.

5. Torok E, Moran E, Cooke F. Oxford Handbook of Infectious Diseases and Microbiology. Oxford (UK): Oxford University Press; 2009. 
6. Zoorob R, Sidani MA, Fremont RD, et al. Antibiotic use in acute upper respiratory tract infections. Am Fam Physician 2012;86:817-22.

7. Penicillin allergy FAQ. Milwaukee: American Academy of Allergy, Asthma \& Immunology; reviewed 2020 Sept. 28. Available: www.aaaai.org/conditions-and -treatments/library/allergy-library/penicillin-allergy-faq (accessed 2019 Nov. 21).

8. Turner NA, Moehring R, Sarubbi C, et al. Influence of reported penicillin allergy on mortality in MSSA bacteremia. Open Forum Infect Dis 2018;5: ofy042.

9. Mattingly TJ II, Fulton A, Lumish RA, et al. The cost of self-reported penicillin allergy: a systematic review. 7 Allergy Clin Immunol Pract 2018;6:1649-54.e4.

10. Macy E, Contreras R. Health care use and serious infection prevalence associated with penicillin "allergy" in hospitalized patients: a cohort study. f Allergy Clin Immunol 2014;133:790-6.

11. MacFadden DR, LaDelfa A, Leen J, et al. Impact of reported beta-lactam allergy on inpatient outcomes: a multicenter prospective cohort study. Clin Infect Dis 2016;63:904-10.

12. Sundquist BK, Bowen BJ, Otabor U, et al. Proactive penicillin allergy testing in primary care patients labeled as allergic: outcomes and barriers. Postgrad Med 2017;129:915-20.

13. Harandian F, Pham D, Ben-Shoshan M. Positive penicillin allergy testing results: a systematic review and meta-analysis of papers published from 2010 through 2015. Postgrad Med 2016;128:557-62.

14. Iammatteo M, Alvarez Arango S, Ferastraoaru D, et al. Safety and outcomes of oral graded challenges to amoxicillin without prior skin testing. 7 Allergy Clin Immunol Pract 2019;7:236-43.

15. American Heart Association. Advanced cardiovascular life support (ACLS): provider manual. 16th ed. Sterling (IL): Channing Bete Company, Inc.; 2016.

16. Ibáñez MD, Rodríguez Del Río P, Lasa EM, et al. Penicillin Allergy in Children (APENIN) Task Force; Pediatric Allergy Committee, Spanish Society of Allergy and Clinical Immunology (SEAIC). Prospective assessment of diagnostic tests for pediatric penicillin allergy. Ann Allergy Asthma Immunol 2018;121:235-44.e3.

17. Hjortlund J, Mortz CG, Skov PS, et al. One-week oral challenge with penicillin in diagnosis of penicillin allergy. Acta Derm Venereol 2012;92:307-12.

18. Labrosse R, Paradis L, Lacombe-Barrios J, et al. Efficacy and safety of 5-day challenge for the evaluation of nonsevere amoxicillin allergy in children. 7 Allergy Clin Immunol Pract 2018;6:1673-80.

19. Drug allergies. Arlington Heights (IL): American College of Allergy, Asthma and Immunology. Available: https://acaai.org/allergies/types/drug-allergies (accessed 2017 Nov. 16).

20. Cox L, Larenas-Linnemann D, Lockey RF, et al. Speaking the same language: the World Allergy Organization Subcutaneous Immunotherapy Systemic Reaction Grading System. 7 Allergy Clin Immunol 2010;125:569-74, 574.e1-7.

21. Mirakian R, Leech SC, Krishna MT, et al.; Standards of Care Committee of the British Society for Allergy and Clinical Immunology. Management of allergy to penicillins and other beta-lactams. Clin Exp Allergy 2015; 45:300-27.

22. James C, Bernstein DI. Allergen immunotherapy: an updated review of safety. Curr Opin Allergy Clin Immunol 2017;17:55-9.

23. Caminati M, Dama AR, Djuric I, et al. Incidence and risk factors for subcutaneous immunotherapy anaphylaxis: the optimization of safety. Expert Rev Clin Immunol 2015;11:233-45.
24. Kuruvilla M, Shih J, Patel K, et al. Direct oral amoxicillin challenge without preliminary skin testing in adult patients with allergy and at low risk with reported penicillin allergy. Allergy Astbma Proc 2019;40:57-61.

25. Savic L, Gurr L, Kaura V, et al. Penicillin allergy de-labelling ahead of elective surgery: feasibility and barriers. Br 7 Anaesth 2019;123:e110-6.

26. Huang K-HG, Cluzet V, Hamilton K, et al. The impact of reported beta-lactam allergy in hospitalized patients with hematologic malignancies requiring antibiotics. Clin Infect Dis 2018;67:27-33.

Affiliations: Department of Family Medicine (Gateman), McMaster University, Kitchener, Ont.; Department of Family Medicine (Gateman), Western University, Kitchener, Ont.; North Perth Family Health Team (Rumble), Listowel, Ont.; Departments of Pediatrics and Child Health, and Food and Human Nutritional Sciences (Protudjer), University of Manitoba; George \& Fay Yee Centre for Healthcare Innovation (Protudjer); The Children's Hospital Research Institute of Manitoba (Protudjer), Winnipeg, Man.; Department of Medicine (Kim), Western University; Department of Medicine (Kim), McMaster University, Kitchener, Ont.

Contributors: Derek Gateman contributed to the conception and design of the study, collection of data, drafting and editing of the work. Jessie Rumble contributed to the collection of data, as well as drafting and editing of the work. Jennifer Protudjer contributed to the analysis of the data for the work and drafted the results section. Harold Kim contributed to the conception and design of the study and edited the final manuscript. All authors gave final approval of the version to be published and agreed to be accountable for all aspects of the work.

Funding: No funding was received for this study.

Content licence: This is an Open Access article distributed in accordance with the terms of the Creative Commons Attribution (CCBYNCND 4.0) licence, which permits use, distribution and reproduction in any medium, provided that the original publication is properly cited, the use is noncommercial (i.e., research or educational use), and no modifications or adaptations are made. See: https://creativecommons.org/ licenses/by-nc-nd/4.0/

Data sharing: Individual participant data that underlie the results reports, once de-identified, may be requested beginning 3 months and ending 5 years following article publication by researchers who provide a methodologically sound proposal to meet the aims in the approved proposal. Proposals should be directed to derek.gateman@ medportal.ca. To gain access, data requestors will be required to sign a data access agreement.

Supplemental information: For reviewer comments and the original submission of this manuscript, please see www.cmajopen.ca/content/9/2/ E394/suppl/DC1. 